\title{
A review of experimental procedures of gas chromatography-mass spectrometry (gc-ms) and possible sources of analytical errors
}

\author{
Waheed Gbenga Akande \\ Department of Geology, Federal University of Technology, Minna, Nigeria
}

Email adress:

geowaheed2008@yahoo.com (W. G. Akande)

\section{To cite this article:}

Waheed Gbenga Akande. A Review of Experimental Procedures of Gas Chromatography-Mass Spectrometry (GC-MS) and Possible Sources of Analytical Errors, Earth Science. Vol. 1, No. 1, 2012, pp. 1-9. doi: 10.11648/j.earth.20120101.11

\begin{abstract}
This paper reviews the experimental procedures of the Gas Chromatography-Mass Spectrometry (GC-MS) technique. The objectives of this study are to present a step-by-step procedure involved in the GC-MS analysis and interpretations of the resulting data both qualitatively and quantitatively, and to identify the possible sources of analytical errors that may be encountered by young inexperienced organic geochemists. The overall process encompasses ultrasonic solvent extraction, fractionation and GC-MS analysis itself. The qualitative and quantitative information obtained from the GC-MS interpretations when integrated together is capable of giving us an insight into the source or origin, depositional environment and level thermal maturity of the analysed sample. The possible sources of analytical errors may include contamination, measurement errors, mechanical/instrumental errors, fractionation errors, and loading errors on the GC. Analytical geochemists must therefore be aware of these errors among others in order to minimise them to the reasonable level. It is recommended that, if weak signals result from the GC-MS analysis of a fraction, such a fraction should be prepared in higher concentration and re-run using selected ion monitoring (SIM) mode so as to enhance signal to noise ratio, and that the GC-MS data should always be interpreted with GC-MS softwares with biomarkers definitions in order to obtain more reliable and robust interpretations.
\end{abstract}

Keywords: Gas Chromatography-Mass Spectrometry, Bitumen, Biomarker, Ultrasonic Extraction, Fractionation, Analytical Errors

\section{Introduction}

Petroleum source rocks consist of sedimentary organic matter and inorganic matter components. The former encompasses both terrestrial and marine (plant and animal) materials accumulated and preserved in sediments and sedimentary rocks such as shales and carbonates. Characterization of organic matter (quantity, quality, generatingpotential and maturity) in the potential source rocks has always been the interests of petroleum geochemists and exploration teams in the oil and gas industries during source rock studies (or evaluation) and hydrocarbon exploration respectively. Studies have shown that organic matter in a typical source rock such as shale is basically divided into two fractions: kerogen and bitumen [1]. He defined kerogen as the portion of organic matter in sedimentary rocks that is insoluble in organic solvent, and bitumen as that fraction of organic matter which is soluble in organic solvent. Williams [2] and Welte et. al. [3] pointed out that studies of the bitumen fraction are particularly useful in correlating pooled liquid petroleum to its source bed. Dow [1] stressed further that bitumen fraction can also be utilized to estimate the convertibility of organic matter to petroleum when integrating with kerogen studies. Thus, bitumen fraction remains an important source of qualitative and quantitative information for petroleum geochemists and exploration geologists despite its smaller portion in common source rocks (e.g. shale) where it accounts for approximately $10 \%$ of the organic matter compared to kerogen accounting for about $90 \%$ (Nwachukwu, personal communications). Bitumen extraction from sediments and sedimentary source rocks therefore become imperative, and hence the study of its chemical components, biomarkers, by Gas Chromatography - Mass Spectrometry (GC-MS) analysis.

This paper focuses on description of the experimental procedures of Gas Chromatography - Mass Spectrometry 
(GC-MS) analysis via dichloromethane - methanol (DCM/MeOH) ultrasonic extraction and fractionation and the possible sources of analytical errors that young organic geochemists and exploration teams need to be cautious of.

\section{Materials and Methods}

\subsection{DCM/MeOH Ultrasonic Extraction}

$\mathrm{DCM} / \mathrm{MeOH}$ Ultrasonic extraction uses ultrasonic vibrations to extract organic matter from solid rock samples with admixture of dichloromethane $\left(\mathrm{CH}_{2} \mathrm{Cl}_{2}\right)$ and methanol $\left(\mathrm{CH}_{3} \mathrm{OH}\right)$ solvents in the ratio of 93:7 in an ultrasonic bath. Admittedly, there are other methods of bitumen extraction in use in modern laboratories. One of such methods is Soxhlet extraction method where organic components in solid samples are extracted from the matrix by continuously washing the solid with a volatile solvent in a specialised piece of glassware (soxhlet extraction apparatus). However, conventional $\mathrm{DCM} / \mathrm{MeOH}$ ultrasonic extraction method is still in use for chemical extraction of solid samples even in some laboratories of developed countries because its procedures are fairly simple as it will be seen in the later sections of this paper.

\subsubsection{Common Apparatus used in DCM/MeOH Ultrason- ic Extraction}

The kinds or sets of apparatus commonly employed for the $\mathrm{DCM} / \mathrm{MeOH}$ ultrasonic extraction method are not too different from those we do find in inorganic geochemistry laboratories. Some of the relevant apparatuses are as shown in Figure 1 and functions of some of these are summarised in Table 1. Others include funnel, measuring cylinder, round bottom flask, test-tube rack, vials (various sizes and shapes), pipette, aluminium foil (tin foil often used as wrappers), hand gloves, spatula and rotary evaporator, just to mention a few.

Table 1. Functions of some relevant apparatus commonly used in ultrasonic extraction.

\begin{tabular}{|c|c|c|}
\hline Label & Name of the apparatus & Functions \\
\hline a & Dirty drying oven & Used to dry things like wet rock samples at $110^{\circ} \mathrm{C}$. \\
\hline b & Clean drying oven & Used to dry clean items such as glasswares at $110^{\circ} \mathrm{C}$. \\
\hline c & Weighing machine & Used to take measurement of rock samples, glasswares, extracts, etc. \\
\hline d & Pestle and mortar & Used for crushing and homogenisation of the rock samples. \\
\hline $\mathbf{e}$ & Centrifuge & $\begin{array}{l}\text { Used for spinning the solid-solvent mixture, concentrating the pellets and separating the super- } \\
\text { natants as }{ }^{*} \text { EOM. }\end{array}$ \\
\hline f & Ultrasonic bath & Used for extracting the soluble organic matter component from pulverised rock samples. \\
\hline g & Ultra Pure Water machine & Used to produce clean, deionised water at $18.2 \Omega \mathrm{m}$. \\
\hline h & Evaporator (Nitrogen-steaming apparatus) & Used for concentrating supernatant by reducing its volume under a stream of nitrogen. \\
\hline i & Capping tool & Used for putting a cover/cap on small vials containing extract fractions. \\
\hline j & Decapping tool (Decapper) & Used for removing a cover/cap on small vials previously used for extract fractions. \\
\hline k & Working bench & A table/bench allocated to a geochemist to use as a workplace in the laboratory. \\
\hline 1 & Test tube & Used for boiling solid-liquid mixtures; to obtain extracts, bitumen fractions, etc. \\
\hline
\end{tabular}

${ }^{*}$ EOM $=$ Extractable organic matter. 


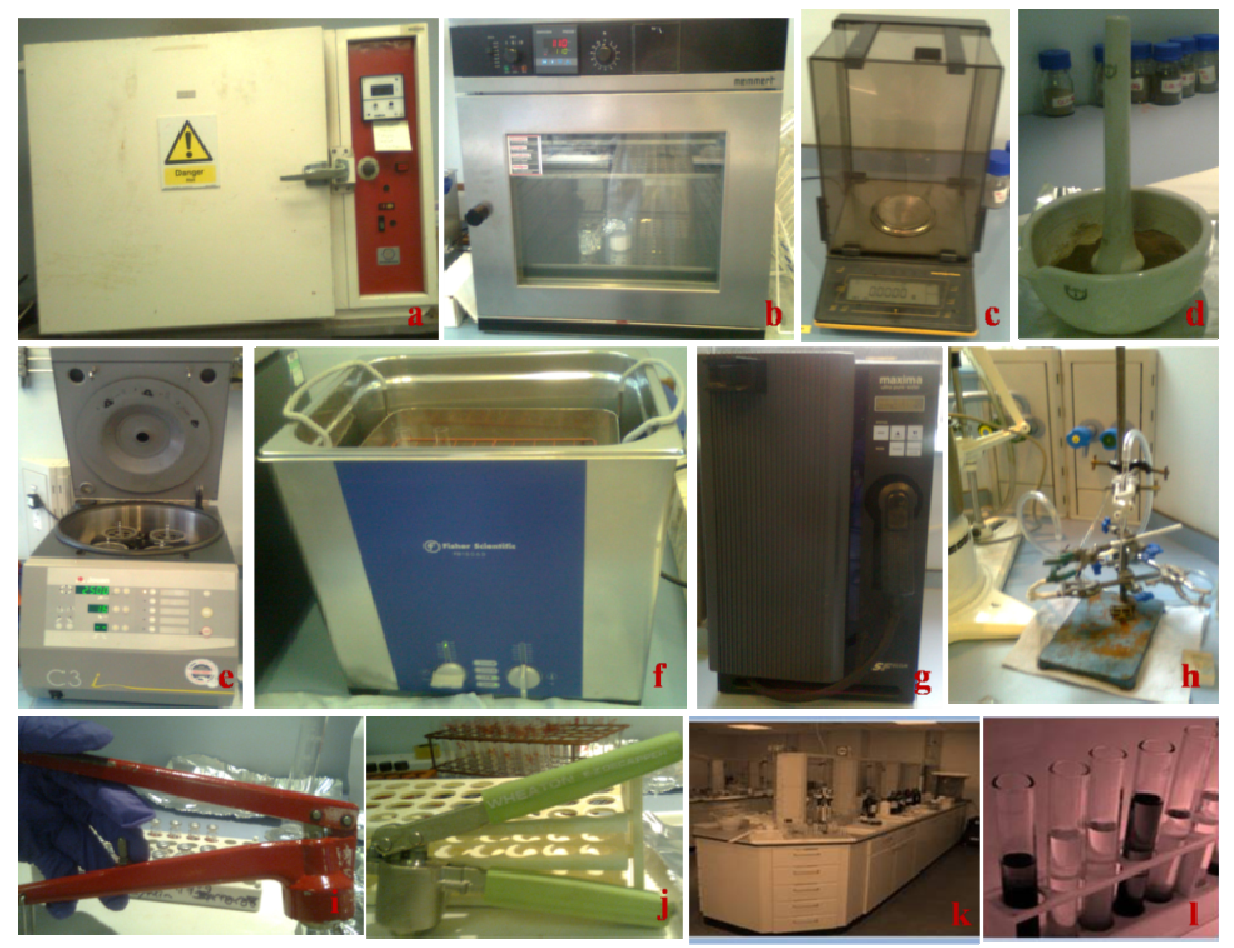

Figure 1. Common Apparatus used in organic geochemistry laboratory for solvent (ultrasonic) extraction. Source: Personal Photos taken at Imperial College Petrophysics/Organic Geochemistry Laboratory in June 2011.

\subsubsection{Common Reagents used in DCM/MeOH Ultra- sonic Extraction}

As the name implies, the chief reagents in DCM / $\mathrm{MeOH}$ ultrasonic extraction are dichloromethane $\left(\mathrm{CH}_{2} \mathrm{Cl}_{2}\right)$ and methanol $\left(\mathrm{CH}_{3} \mathrm{OH}\right)$. Other reagents in use include the internal and external standards which are usually used as Quality Control check and for quantification purposes in the subsequent analyses. Common internal standards are adamantane, squalane, hexamethylbenzene and p-terphenyl.

\subsection{Extraction Process}

The DCM/MeOH ultrasonic extraction method usually starts with sample preparation. If the rock samples collected from the field are dirty or contain suspected extraneous materials, such samples may be washed or its surface be scrapped to remove the dirty portions or materials. The samples can then be wrapped in tinfoil and put in drying oven (in this case 'dirty drying oven') at $110^{\circ} \mathrm{C}$ to dry properly. Disaggregation (pulverization) and homogenization of the samples are usually achieved with the aid of pestle and mortar and beware that other mechanical devices may be found in some modern laboratories for the same purpose. A few grams of the pulverized samples (e.g. $2-3 \mathrm{~g}$ ) are usually weighed into a test-tube using a weighing machine. The centrifuge works on the principles of sedimentation where the centripetal acceleration is used to separate substances of density contrast and on the concept of settling velocity as predicted by Stokes' law. This way, gravitational force caused the sol- id and denser part to precipitate and gather at the bottom of the test-tube while the supernatant, the solution containing organic matter stays on top.

After centrifugation, the supernatant solution needs to be filtered off from the test-tube by using a pipette (e.g. a pipette in the form of a rubber dropper), without disturbing the pellet. After filtration, the aliquot of the solvent mixture is to be added to the pellet (residue) in the testtube to the half-way as before and the whole procedures have to be repeated for the second time, and for the third time for each sample. The filtered supernatants are to be concentrated by evaporation either by using rotary evaporator or under a stream of nitrogen. The concentrated fractions then need to be transferred into clean, empty, pre-weighed vials, evaporated further to reduce the volume of the fraction to less than $1 \mathrm{ml}$, and left to completely evaporate off overnight so as to obtain the mass of dry extracted organic matter (rock powder).

The yield, the mass of extractable organic matter (EOM) expressed as $\mathrm{mg}$ of extract / $\mathrm{g}$ of rock (or as a percentage) is obtained as follows.

$$
\begin{aligned}
\text { Extract weight } & =\text { vial }+ \text { extract } \\
& - \text { clean vial }
\end{aligned}
$$

Percentage extractable

$$
\begin{aligned}
& \text { organic matter }(\% \mathrm{EOM})= \\
& \frac{\text { Extractweight }}{\text { Massofsamplebeforeextraction }}
\end{aligned}
$$


The analytical results of the EOM may be presented in table form as shown in Table 2.

Table 2. A typical representation of extracted organic matter (EOM) results from $\mathrm{DCM} / \mathrm{MeOH}$ solvent (ultrasonic) extraction.

\begin{tabular}{llll}
\hline $\begin{array}{l}\text { Sample/ } \\
\text { Formation }\end{array}$ & $\begin{array}{l}\text { Mass of sample } \\
\text { before extraction } \\
(\mathbf{g})\end{array}$ & $\begin{array}{l}\text { Mass of dry ex- } \\
\text { tracted rock } \\
\text { powder }(\mathbf{g})\end{array}$ & $\begin{array}{l}\text { EOM (mg of } \\
\text { extract/g of } \\
\text { rock) }\end{array}$ \\
\hline $\begin{array}{l}\text { Blue Lias } \\
\text { Kimmeridge }\end{array}$ & 2.4950 & 0.0075 & 3.01 \\
Clay & 2.6831 & 0.0027 & 1.01 \\
Kimmeridge & 2.7872 & 0.0126 & 4.52 \\
Clay & 2.5306 & 0.0007 & 0.28 \\
Gault Clay & 2.7124 & 0.0006 & 0.22 \\
Nothe Clay & 2.7611 & 0.0014 & 0.51 \\
Oxford Clay & 2.76 & 1.04 \\
Oxford Clay & 2.7901 & 0.0029 & 1.93 \\
Purbeck & 2.6361 & 0.0051 & \\
Black Shale & & &
\end{tabular}

Source: Akande [4].2.3. Sulphur Removal

For marine source rocks especially those that been described to contain Type II kerogen, sulphur removal from the extract might be very necessary. Elemental sulphur may be removed prior to subsequent analyses such as fractionation (column chromatographic separation) and Gas Chromatography-Mass Spectrometry (GC-MS) analyses so as to avoid interference of sulphur with other compounds of interest. The steps for sulphur removal as set out in Imperial Lab Manual (2009) are as follows:

Copper Activation

Place copper turnings in $10 \% \mathrm{HCl}$ for a few hours

Remove activated copper

Wash with RO water and decant

Store activated copper under solvent (usually hexane)

Reactivate when shiny surface becomes dull

Sample Treatment

Dilute extract with solvent (93:7 v/v DCM/MeOH) in small vial

Place activated copper turnings in vial and leave overnight

If copper turns black replace with new activated copper

If any copper remains shiny reaction is complete

Thus, shininess of copper grains is an indication that the sample is sulphur free. The typical work flowchart for the ultrasonic extraction and the subsequent analyses is as shown in Figure 2.

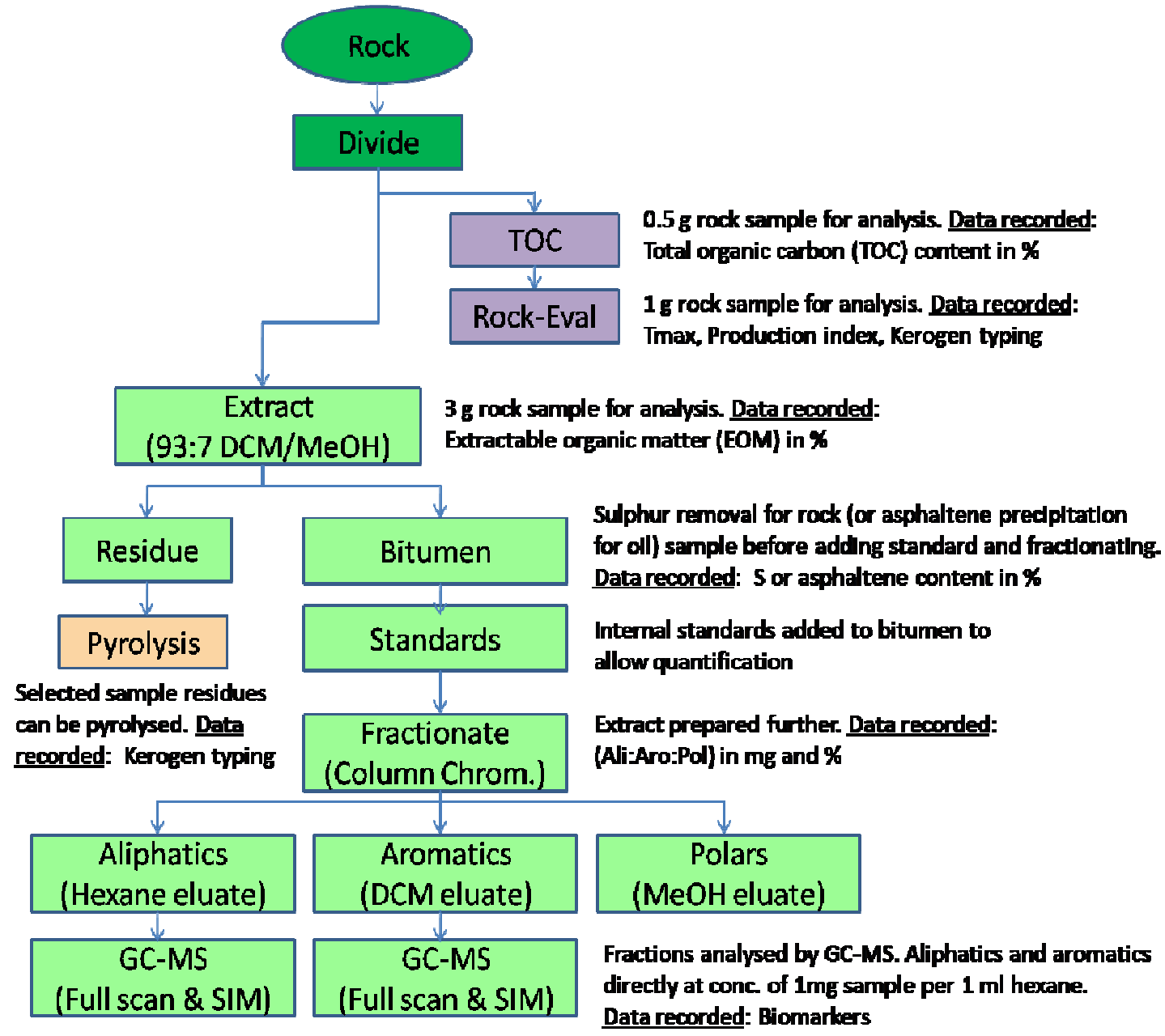

Figure 2. A typical work flowchart in ultrasonic extraction process and the subsequent analyses. Green boxes are routine and other coloured boxes represent optional extra. (Modified after [5]). 


\subsection{Column Chromatographic Separation (Fractiona- tion)}

Following DCM/MeOH ultrasonic extraction, the samples are usually fractionated on the column chromatography (Figure 3) using silica gel (or alumina) which was activated at a temperature greater than $110^{\circ} \mathrm{C}$ for approximately an hour as the solid phase, and three different solvents - hexane $\left(\mathrm{C}_{6} \mathrm{H}_{14}\right)$, dichloromethane (DCM), $\mathrm{CH}_{2} \mathrm{Cl}_{2}$ and methanol $(\mathrm{MeOH}), \mathrm{CH}_{3} \mathrm{OH}$ as the mobile phase. The column (Figure 3 ) is to be prepared by plugging the lower part of the column with small amount of pre-extracted quartz wool (or glass wool), and by rinsing with non-polar solvent mixture. This step is to be followed by addition of activated silica gel (or alumina) to the column to the required volume. Finally, extractable organic matter of the sample to be fractionated has to be placed on top of the column.

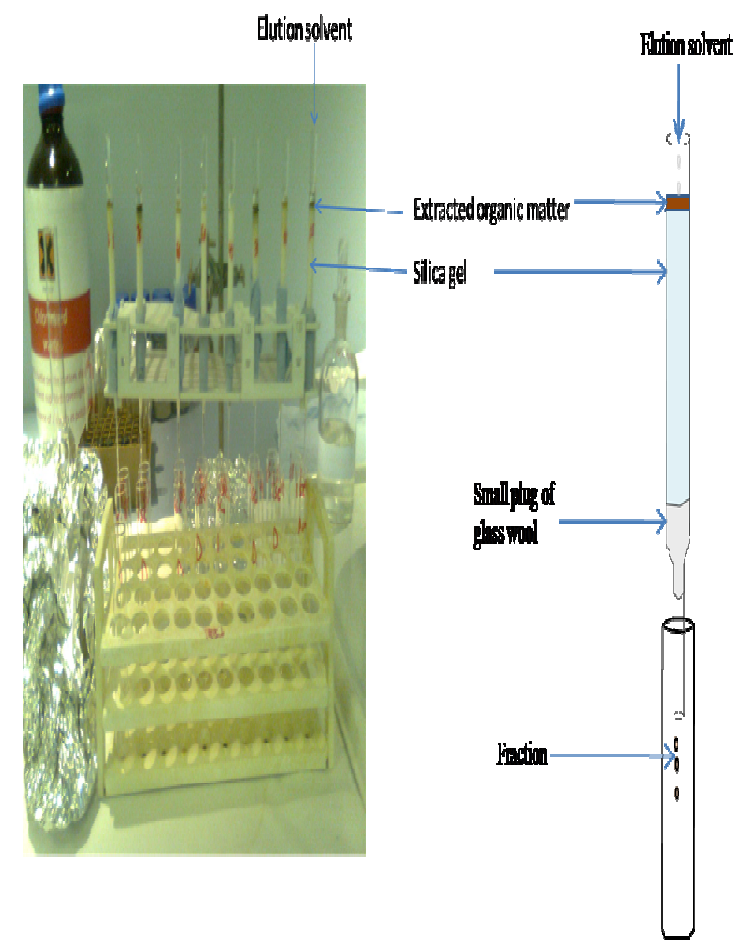

Figure 3. Column Chromatography set up for separation of aliphatic, aromatic and polar fractions.

The column needs to be eluted with three different solvents to give three different fractions: $3 \mathrm{ml}$ of hexane $\left(\mathrm{C}_{6} \mathrm{H}_{14}\right), 3 \mathrm{ml}$ of dichloromethane $\left(\mathrm{CH}_{2} \mathrm{Cl}_{2}\right)$, and $3 \mathrm{ml}$ of methanol $\left(\mathrm{CH}_{3} \mathrm{OH}\right)$ in this in order, and the fractions are collected in three separate clean, pre-weighed test-tubes. The first fraction contains aliphatic (straight- and branched-chained) hydrocarbons; the second fraction contains aromatic (ring-containing) hydrocarbons; and the third fraction contains polar fraction which is rich in nitrogen, sulphur and oxygen (NSO) compounds. The three fractions have to be concentrated under a stream of nitrogen and left to completely dry off in order to obtain their masses. The normalised percentages composition of three fractions can be displayed on aliphatic-aromaticpolar fraction ternary diagram. Such a diagram is useful in the determining the level of thermal maturity of the organic matter and degree of biodegradation of the sample.

For quality control check and quantification purposes, the internal standards consisting of $46 \mu \mathrm{g}$ of adamantane and $40 \mu \mathrm{g}$ of squalane in hexane, and $38.8 \mu \mathrm{g}$ of hexamethylbenzene and $39.2 \mu \mathrm{g}$ of p-terphenyl in hexane, for instance, may be prepared; and about $100 \mu \mathrm{l}$ of the former and the latter can be added to the aliphatic and aromatic fractions respectively prior to the Gas Chromatography - Mass Spectrometry (GC-MS) analysis. Alternatively, the internal standards may be added just before fractionation (Figure 2).

\subsection{Gas Chromatography - Mass Spectrometry (GC- MS) Analysis}

After fractionation, saturate and aromatic fractions often receive most attention for additional analysis. In contrast, in spite of their possession of useful information, polar fractions containing nitrogen sulphur and oxygen (NSO) compounds often suffer from further analysis due to their complexity. Thus, aliphatic and aromatic fractions are usually selected for a GC-MS analysis. Meanwhile, if the interest lies in the polar fraction, the fraction may be subjected to asphaltene precipitation using $n$ pentane so as to obtain maltene fraction (asphaltene-free fraction) which will be subsequently used for bulk-group analysis by TLC-FID (latroscan) and for preparative group separation by MPLC [6].

Just before the analysis, aliphatic and aromatic fractions are to be dissolved in hexane and DCM respectively, in order of $1000 \mu \mathrm{l}$, in separate vials. From each vial, 1 $\mu \mathrm{l}$ of solution is to be injected into the column by the GC-MS machine, for example, Agilent DB-5 column which comprises an Agilent $6890 \mathrm{~N}$ gas chromatograph (Figure 4). The GC is usually coupled to a Mass Spectrometer (detector), for example, a 5973 Mass Selective Detector (MSD) which has the onus function of recording the mass spectrum of the chemical compounds as they come out of the $\mathrm{GC}$ and after fragmentation processes by a stream of electrons in the mass spec. Helium gas is often used a carrier gas, although other gases like nitrogen may also be used. It is recommended by NIGOGA [6] that the GC oven should be initially held at $80^{\circ} \mathrm{C}$ for 1 minute, and then warmed at $4-6^{\circ} \mathrm{C} \mathrm{min}^{-1}$ to about $300^{\circ} \mathrm{C}$, where it is to be held for 20 minutes, and the total length of time for running the analysis may be determined and programmed by the analytical geochemist (GC-MS analyst). Peaks in the chromatograms produced by these analyses were identified by a combination of references to their mass spectra and the NIST08 mass spectral database, and by comparing retention times and elution orders with those of known standards previously analysed on the same GC-MS. 


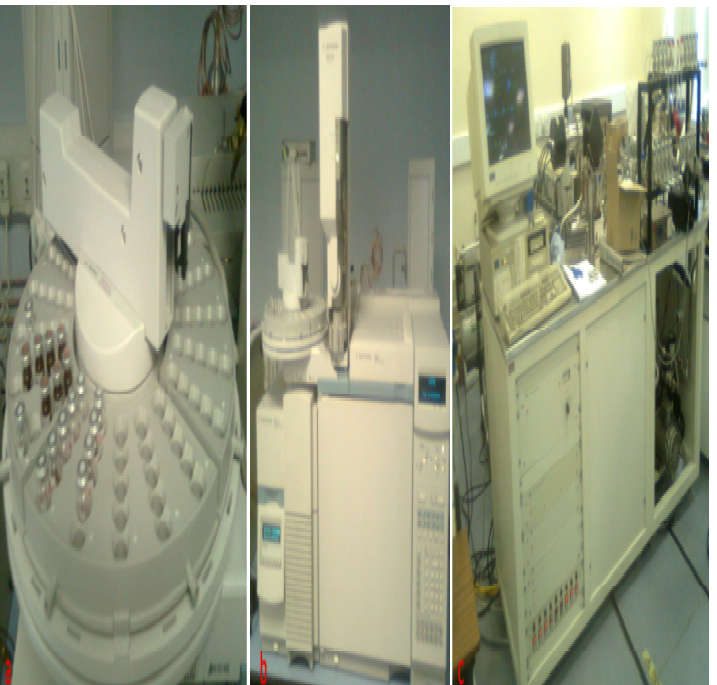

Figure 4. GC-MS set up. a-shows the compartment tray for loading the samples (in capped vials), $b$ - shows the column to the centre/right coupled to a Mass Spectrometer to the left, and c-shows a desktop for programming the analysis and recording the results (chromatograms and mass spectra).

Due to advancement in technology, different versions of the columns (gas chromatograph) and Mass Spectrometer can now be found in the modern laboratories. However, the operational procedures and conditions such as oven temperature program, column type and carrier gas are often similar in different GC-MS models. This does not mean that the retention time (the time at which a chemical compound comes out the GC and detected on the MS) at which a particular compound is detected in one laboratory should be exactly the same in another laboratory.

It is of particular note that the choice of the GC-MS model depends on the research problem a researcher (geochemist, environmentalist, pharmacist, etc.) is trying to answer. The analyses can be run in different modes such as full scan, Total Ion Current (TIC), and Selected Ion Monitoring (SIM) modes to enhance signal-to-noise ratio for weak peaks.

\section{Results and Discussion}

\subsection{Interpretations of GC-MS Results}

As earlier alluded, the GC-MS results or data can be interpreted both qualitatively and quantitatively depending upon the interests of the researchers.

\subsection{Qualitative Interpretations}

Qualitative interpretation involves all series of tasks of studying, identifying, naming and classification of chemical compounds of the analysed mixtures. Chemical compounds can be identified on the basis of their mass spectrum, which is basically a unique fingerprint for each molecule. This fingerprint can then be utilized to identify unknown compounds. For instance, the mass spectrum displayed in Figure 6 was produced by dodecane, $\mathrm{C}_{12} \mathrm{H}_{26}$, represented the first peak at retention time of $4.97 \mathrm{mi}$ nutes on the chromatogram shown in Figure 5.

\section{TIC: CHECKOUT.D}

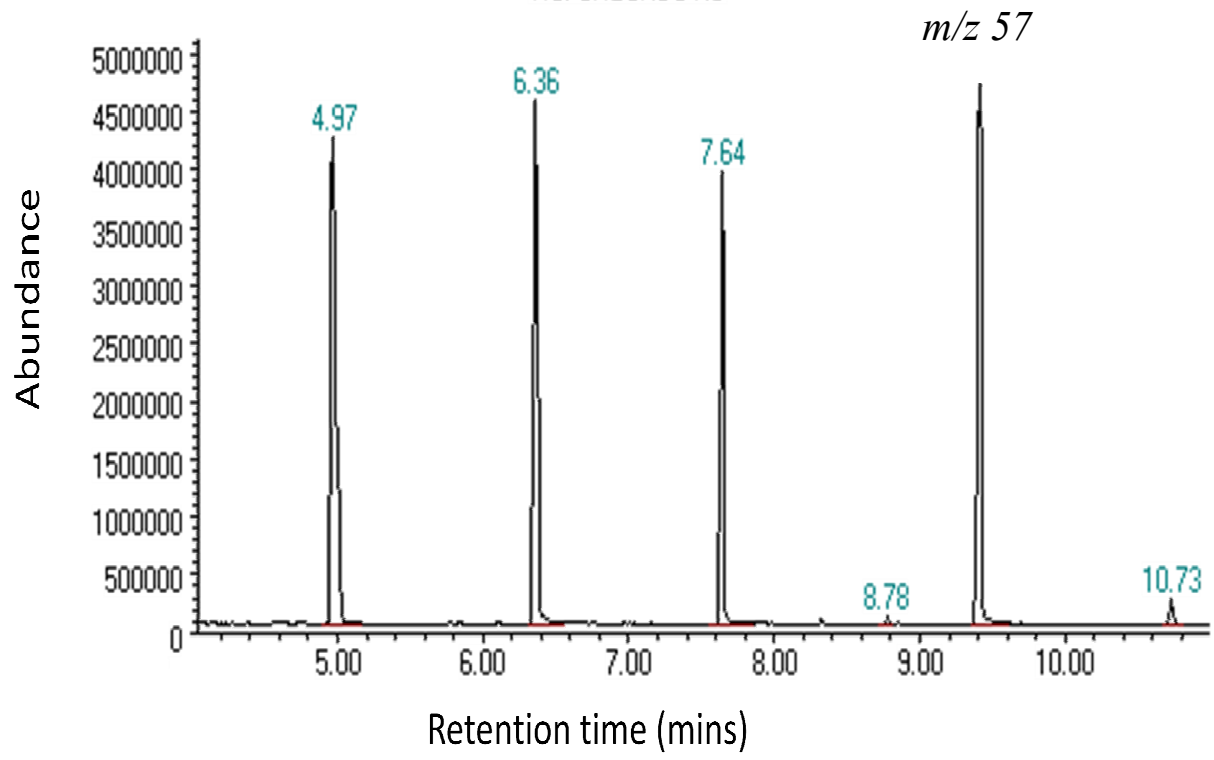

Figure 5. Chromatogram generated by a $G C$. 


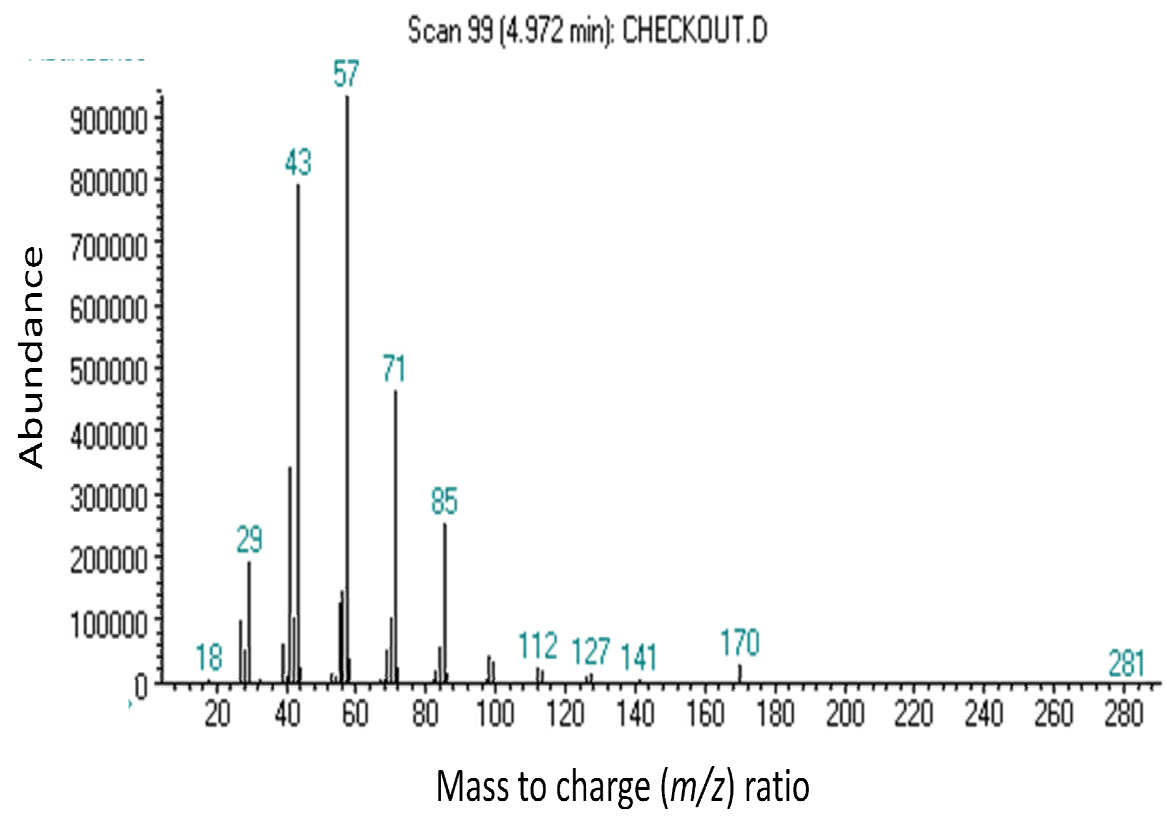

Figure 6. Mass-spectrum generated by an MS.

The computer on the GC-MS has a library of spectra that can be used to identify an unknown chemical compound in the sample mixture. The library compares the mass spectrum from a sample component to mass spectra in the library and reports a list of likely identifications (including names and possible molecular formulae and structures) along with the statistical probability of the match. Some of the possible compounds that can be identified in aliphatic (saturate) fraction are normal alkanes ( $n$-alkanes), isoprenoids (pristane and phytane), hopanes and steranes. The peak heights for normal alkanes (e.g. dodecane) shown on chromatogram in Figure 5 are obtained from the mass to charge $(\mathrm{m} / \mathrm{z})$ ratio of 57 , $\mathrm{m} / \mathrm{z}$ 57. Isoprenoids are also obtained from the same $\mathrm{m} / \mathrm{z}$ 57 while the hopanes (triterpanes) and steranes are obtained from $\mathrm{m} / \mathrm{z} 191$ and 217 respectively. In the same vein, aromatic compounds in aromatic fractions such as carotenoids can be obtained from $\mathrm{m} / \mathrm{z}$ 133. These biomarkers furnish us with useful pieces of information on the source and origin of the organic matter, environment of deposition and the level of thermal maturity, and these have been described in details by earlier workers [7]; [8]; [9]; [10]; [11]; [12]; [13]; [14].

\subsubsection{Quantitative Interpretations}

Although it not very common in the literature like qualitative interpretations, chemical compounds identified from the GC-MS results can also be expressed or reported in quantitative terms. In fact, this the main rationale behind the use of internal standards as earlier mentioned. Like any other unknown compounds on the chromatogram, the internal standards also have their peaks (peak areas) which form the basis for quantitative interpretations.

Assuming that the concentration of the internal stan- dard added to the sample mixture is denoted by $\mathrm{C}_{i}$, and the peak areas of internal standard and the unknown compound are represented by $\mathrm{A}_{i}$ and $\mathrm{A}_{c}$ respectively. Then, the concentration of the unknown compound, $\mathrm{C}_{c}$ can be obtained from the relation below.

$$
C_{c}=\frac{A_{i}}{A_{c}} X C_{i}
$$

The peak (area) ratio, $\mathrm{A}_{i} / \mathrm{A}_{c}$, can be evaluated by using 'Integration Results' mode of the GC-MS software. The concentration of the identified unknown compound may be expressed in the unit of concentration of the internal standard (e.g. mg/l) or may be converted using appropriate conversion factors to other units of concentration such as $\mathrm{mg} / \mathrm{ml}, \mu \mathrm{g} / \mathrm{ml}, \mu \mathrm{g} / \mu \mathrm{l}$, parts per million (ppm), parts per billion (ppb), etc. The quantitative information obtained on the relative abundances of biomarkers in a sample when integrated with qualitative interpretations can be used to characterise the source, depositional settings and level of thermal maturation of the sample.

\subsection{Possible Sources of Analytical Errors in DCM/MeOH Ultrasonic Extraction, Fractionation and Gas Chromatography-Mass Spectrometry Analysis}

The DCM/MeOH ultrasonic extraction, like any other extraction methods, is very crucial to our study of potential source rocks, and of course biomarker studies. In the light of this, every errors made in the process will be automatically transferred to the subsequent analysis of the bitumen to its aliphatic, aromatic and polar fractions (fractionation), and ultimately to the Gas Chromatography-Mass Spectrometry (GC-MS) analysis. It has therefore become very imperative to identify where errors may be ensued in the course of carrying out the extrac- 
tion processes, fractionation and Gas ChromatographyMass Spectrometry (GC-MS) analysis in order to safeguard them as much as possible. Some of the possible sources of errors that young or inexperienced organic geochemists may encounter are discussed in this section.

\subsubsection{Contamination}

Contamination has been a serious issue in both organic and inorganic geochemical analyses. This is because contaminants can be introduced into the samples under analysis at any stage of the analysis. The sources of contaminations include using unclean glasswares such as testtubes, funnel, vials; leaving the samples uncovered for a long period of time; taking an object over the sample under analysis; just to mention a few.

Cross contamination is also a common source of contamination. As the name implies, these are the errors introduced into the samples between two successful samples under analysis as a result of not keeping to good or standard laboratory practices such as washing the apparatus properly (and rinse with the appropriate reagents/solvents where necessary) before the next sample is picked. All these contamination sources and others must be safeguarded as a lean or poor sample, in quantitative terms, may give anomalous values which have neither geochemical nor geological significance or interpretations but a mere geochemical artefact.

\subsubsection{Measurement Errors}

Errors often arise in the course of taking measurements in geochemistry laboratory and most of these errors can be ascribed to the sensitivity of the measuring instrument or device, calibration problem, equipment balancing, and inexperience on the part of the experimentalist. For instance, variations in the balancing state (equilibrium position) of the measuring instruments like weighing machine when taking measurement of the same or similar variable at different times may bring about measurement errors. Of particular note is that the vast majority of these errors are usually systematic. Besides, errors may also be introduced in measurement of volumes especially when air is sucked with the reagents when using 'sucker' or pipette or rubber dropper, or when the meniscus is read wrongly. This air-in-thereagent error often reduces the concentration or volume of the interested reagent, and thus may affect the target results.

\subsubsection{Mechanical (Instrumental) Errors}

Sometimes there may be errors arising solely from malfunctioning of the instruments in use which may be difficult for inexperienced geochemist to envisage in advance. This error may be exemplified by a reference to water in the ultrasonic water bath that has become so extremely hot that the boiling solid-solvent mixture starts spilling into the bath. A similar example is also often encountered when the loaded test-tubes are placed unevenly in the centrifuge. In the event, the centrifuge be- comes unbalanced and ceases to spin which may ultimately lead to breaking of the test-tubes. The former example may lead to reduction in the content of the testtube, and hence underestimation of the extract yields and the latter may lead to complete loss of the sample.

\subsubsection{Errors in Fractions Separation (Fractionation)}

It is not uncommon to get one fraction into the other, e.g. aliphatic fraction may get into the aromatic fraction and vice versa, during fractionation; and this makes compounds identification a strenuous exercise during chromatograms interpretations. This error may arise as result of not preparing the column properly, elution with excess volume of the reagent (elution solvent) or due to given improper attention to fraction leaching.

\subsubsection{Loading Errors on the GC}

The loading errors which include improper labelling of the samples or improper matching of the labels on the sample vials with those sample details programmed on the desktop connected to the MS often creates big problems or confusions especially when samples from two or more researchers are co-run together. Besides, contaminations in another dimension can also be introduced on the GC. To address this error, a vial filled with DCM is usually placed in position 1 on the GC tray (Figure 4a) and the idea is that DCM cleanses the column in preparation for the real sample analysis.

\section{Conclusions}

The experimental procedures for the GC-MS involve bitumen extraction, fractionation and ultimately the GCMS analyses. The following points are worth noting:

Conventional $\mathrm{DCM} / \mathrm{MeOH}$ ultrasonic extraction is still common in use today even in the modern laboratories because its procedures are fairly simple, relatively cheaper to run and it is faster compared to other extraction methods like soxhlet extraction method.

Extracted organic matter (EOM) is a complex mixture of various organic compounds, and it must be separated into its component fractions: aliphatic, aromatic and polar fractions.

The GC-MS machine is versatile in its applications. Besides being commonly employed to study biomarkers in soluble bitumen fractions, it also finds application in other areas such as the medical, pharmacological, environmental, chemical engineering, law enforcement fields, etc. Thus, in geochemical context, individual components or compounds in the soluble extracts or oil samples can be studied or identified by utilising the GC-MS machine.

The GC-MS results or data can be interpreted both qualitatively and quantitatively, and these interpretations provide crucial information on the source, depositional environment and level of thermal maturity of the sample.

The overall processes of analysing chemical components or compounds by GC-MS via ultrasonic extraction 
and fractionation are not without errors such as contamination, measurement errors, mechanical/instrumental errors, fractionation errors, loading errors on the GC, etc. and analytical geochemists must be aware of these errors among others in order to minimise them to the reasonable level.

\section{Recommendations}

It is recommended that the young or inexperienced geochemists should always keep to the standard laboratory (experimental) practices as this will enable them to obtain a more reliable result which will translate to writing up their reports easily and successfully within the shortest possible time and save the time of returning back to the laboratory to repeat their experiments. For easy detection of errors especially contamination, which is a huge issue in geochemistry, blank samples should always be run together with the actual sample, or alternatively the blanks should be run just before or after the analysis. The fractions overlap during the fractionation process should be monitored with the ultra-violet (UV) light in order to prevent one fraction getting into the other. Additionally, for weak signals resulting from the GC-MS analyses, the fraction under analysis should be prepared in higher concentration and re-run using selected ion monitoring (SIM) mode so as to enhance signal to noise ratio, and to obtain more reliable and robust interpretations. Finally, the GC-MS data should always be interpreted with GC-MS softwares with biomarkers definitions.

\section{References}

[1] W. G. Dow, "Kerogen Studies and Geological Interpretations. Journal of Geochemical Exploration", vol. 7, pp. 77-79, 1977.

[2] J. A. Williams, "Characterization of oil types in Wiliston Basin", American Association Petroleum Geologists Bulletin, vol. 58, pp. 1243-1252, 1974.

[3] D. H. Welte, H. W. Hagemann, A. Hollerbach, and D. Leythanuser, "Correlation between petroleum and source rocks", Proceedings $9^{\text {th }}$ World Petroleum Congress, vol. 2: pp. 179-191, 1976.

[4] W. G. Akande, "Mesozoic Organic-rich Rocks of Southern England", Unpublished M.Sc. Dissertation. Imperial College, London, 2011.

[5] Imperial Lab Manual, Imperial College Petrophysics/Organic Laboratory Manual Guide for 2009/2000 academic session, 2009.

[6] NIGOGA, The Norwegian Industry Guide to Organic Geochemical Analyses, Edition 4.0. Published by Norsk Hydro Statoil Geolab Nor SINTEF Petroleum Research Norwegian Petroleum Directorate, 2000.

[7] B. M. Didyk, B. R. T. Simoneit, S. C. Brassell, and G. Eglinton, "Organic geochemical indicators of paleoenvironmental conditions of sedimentation", Nature, vol. 272, pp. $216-222,1978$.

[8] G. W. Lijmbach, "On the Origin of Petroleum, in Proceedings of the 9th World Petroleum Congress", vol. 2. London: Applied Science Publishers, 1975, pp. 57-369.

[9] J. Connan, and A. M. Cassau, "Properties of gas petroleum liquids derived from terrestrial kerogen at various maturation levels", Geochim. Cosmochim.Acta, vol. 44, pp. $10-23,1980$.

[10] K. E. Peters, T. H. Fraser, W. Amris, B. Rustanto, and E. Hermanto, "Geochemistry of crude oils from eastern Indonesia", American Association of Petroleum Geologists Bulletin, vol. 83, pp. 1927 - 1942, 1999.

[11] J. M. Moldowan, W. K. Seifert, and E. J. Gallegos, "Relationship between petroleum composition and depositional environment of petroleum source rocks", American Association of Petroleum Geologists Bulletin, vol. 69, pp. 1255-1268, 1985.

[12] K. E. Peters, and J. M. Moldowan, "Effects of source, thermal maturity, and biodegradationon the distribution and is omerisation of homohopanes in petroleum", Organic Geochemistry, vol. 17, pp. 47-62, 1991.

[13] K. E. Peters, and J. M. Moldowan, The Biomarker Guide: Interpreting Molecular Fossils and Ancient Sediments. Prentice Hall: Eaglewood Cliffs, 1993, pp. 1-363.

[14] P. E. Summons, and T. G. Powell, "Identification of arylisoprenoids in source rock and crude oils: Biological markers for the green sulphur bacteria", Geochimica et Cosmochimica Acta, vol.51, pp. 557-566, 1987. 